\title{
Stratégies d'animation de groupe sur la communication patient-médecin-famille
}

\author{
Richard BOULÉ MD, M.A. - Gilles GIRARD, M. PS. \\ Diane CLAVET, MD, M.A - Carolle BERNIER, MD, M.A.
}

\begin{abstract}
Résumé Contexte : Il s'agit d'une étude qualitative de type recherche action basée sur une analyse réflexive s'échelonnant sur dix ans. But de l'étude : Le but de l'étude est d'explorer des stratégies pédagogiques susceptibles d'optimiser le fonctionnement d'un groupe d'apprentissage aux habiletés de communication patient-médecin-famille. Sujets : Vingt (20) groupes de 10-12 résidents de médecine familiale en stage de six mois à l'UMF-Estrie ont participé à l'étude. Résultats : L'analyse a permis de dégager six catégories de stratégies pédagogiques susceptibles de renforcer les effets positifs des interactions en groupe sur les apprentissages des résidents. Ces six catégories sont : transformer les besoins démontrés en besoins ressentis; amener les résidents à explorer leurs difficultés relationnelles avec les patients; générer le goût d'une intervention guidée par des balises cognitives reconnues; faciliter le passage de la discussion à l'expérimentation concrète; induire l'habitude de l'analyse réflexive en cours d'action; favoriser le transfert des apprentissages en groupe à la pratique clinique. De plus, l'hypothèse suivante a émergé de l'expérience: des stratégies spécifiques sont à privilégier en fonction du type de participants dans chaque groupe. Conclusion : En plus de générer des stratégies d'enseignement en groupe des habiletés de communication patient-médecin-famille, la démarche réflexive sur laquelle s'appuie l'étude, a permis de soutenir la motivation des enseignants en recadrant les difficultés rencontrées en occasions de renouvellement continu de l'enseignement.
\end{abstract}

Mots clés Education médicale, apprentissage en groupe, communication patient médecin.

Summary Context: This qualitative study is based on a reflexive analysis that was developed over a ten-year period. Objective: The purpose of this study is to explore educational strategies that enhance and optimize the positive effects of group dynamics on communication between the patient-doctor-family triad. Subjects: Twenty (20) groups of 10-12 residents within family medicine, doing their six-month rotation at UMF-Estrie, participated in the study. Results: This analysis identified six categories of educational strategies which reinforced the positive effects of group interaction on the residents'learning. These six categories are: transform exhibited needs into those which are felt and integrated; encourage residents to explore any interpersonal difficulties encountered with a patient; develop their desire to adopt interventions guided by specific cognitive markers; facilitate the transition from discussion to concrete experimentation; induce the habit of reflexive analysis during their actual practice; help them transfer group learning to their individual clinical practice. The following hypothesis also emerged from the study : certain specific teaching strategies seem more appropriate and correspond to different participantsstyles. Conclusion: In conclusion, this ongoing reflexive analysis has identified six categories of educational strategies for group learning, on communication between the patient-doctor-family triad. Moreover, it helped to sustain motivation among the faculty by turning difficulties into learning opportunities and to further encourage the learning process.

Key words Medical education, group learning, patient-doctor communication.

Pedagogie Medicale $2000 ; 1: 13-22$.

Département de médecine de famille de l'Université de Sherbrooke et Unité de médecine de famille du CLSC de La RégionSherbrookoise - Faculté de médecine - Université de Sherbrooke - 3001, 12 Avenue Nord - Sherbrooke, Qc J1H 5N4 Canada - Tél. : (819) 346-1110, poste 14276 - Télécopieur : (819) 564-5386 - Courriel : rboule@courrier. usherb. ca 


\section{Recherche et Perspectives}

\section{Introduction}

La communication efficace avec les patients a des impacts démontrés sur divers indicateurs de santé ${ }^{1}$. Son apprentissage constitue un objectif important de la formation en médecine de famille. Des ateliers de formation à la communication avec les patients et leur famille, inspirés de l'approche systémique ${ }^{2}$ et centrée sur le patient, ${ }^{3,4}$ sont offerts aux résidents de plusieurs programmes. La littérature en pédagogie médicale propose peu de modèles d'enseignement en petit groupe de résidents, privilégiant des descriptions d'enseignement au chevet du malade, en clinique externe et par modèle de rôle ${ }^{4}$. Côté et $\mathrm{al}^{5}$ ont récemment décrit la pertinence de divers éléments de cet enseignement basés sur des principes pédagogiques reconnus sans toutefois s'attarder au fonctionnement du groupe. Or, celui-ci influence le degré d'implication et l'apprentissage de ses membres. Le but de l'étude est d'explorer des stratégies pédagogiques susceptibles d'optimiser le fonctionnement d'un groupe d'apprentissage aux habiletés de communication patient-médecin-famille. La démarche réalisée s’inscrit dans les cadres de la recherche action et de l'analyse réflexive.

\section{Méthodologie}

Depuis dix ans, les animateurs d'ateliers hebdomadaires $^{6}$, auxquels participent les dix à douze résidents en stage de six mois à l'Unité de médecine de famille de l'Estrie (UMF), s'impliquent dans une analyse réflexive, c'est-à-dire une autoévaluation et une restructuration continue en cours d'action de leurs stratégies pédagogiques ${ }^{7}$.

Chaque atelier d'une durée de trois heures, animé par une dyade d'enseignants, aborde un des vingt thèmes relatifs à la communication patient-médecin et à l'approche familiale (tableau 1). Différentes formules pédagogiques y sont utilisées ; deux descriptions types d'ateliers illustrent leur agencement (tableau 2). Deux membres de l'équipe d'animation rencontrent individuellement chaque participant au début et à la fin de la série d'ateliers pour discuter de ses objectifs et de son fonctionnement en groupe. A cette occasion, les participants donnent leur accord sur les informations qui seront recueillies auprès d'eux ainsi que sur l'utilisation qui en sera faite pour optimiser cette activité d'apprentissage. Cette démarche s'effectue dans le cadre habituel du processus d'amélioration de l'enseignement.

A chaque édition de la série d'ateliers, les auteurs exami-

\section{Tableau 1 : thèmes d'ateliers}

\section{Communication patient-médecin}

\section{BLOC I - Concepts-clés}

1. Approche globale et négociée.

2. Intimité dans la relation patient-médecin et gestion du stress chez le résident.

\section{BLOC II - Techniques d'entrevue}

3. Début d'entrevue.

4. Suivre un patient pas à pas en entrevue.

5. Fin d'entrevue.

\section{BLOC III - Interactions difficiles}

6. Principes de base en relation d'aide.

7. Patient suicidaire et dépressif.

8. Annonce d'une mauvaise nouvelle.

9. Patient dépendant et psychosomatique.

10. Patient agressif et troubles de la personnalité.

\section{Approche familiale et systémique}

1. Individu en santé et développement adulte.

2. Couple sain et intervention auprès d'un couple.

3. Famille saine et évaluation familiale.

4. Devenir parents.

5. Vivre et parler avec des adolescents.

6. Divorces, familles monoparentales et reconstituées.

7. Violence familiale.

8. Stress, maladie et famille.

9. Vieillissement, retraite et attitudes face à la mort.

10. Le médecin et sa famille. 


\section{Tableau 2 : déroulement type d'atelier}

\section{EXEMPLE 1 \\ Thème : principes de base en relation d'aide}

- Explication du thème et des limites du rôle du médecin en relation d'aide :

- exposé de quelques exemples cliniques.

- Comment aider le patient qui réclame une inter-vention sans être en mesure de se mobiliser pour un tel travail interne?

- questions-discussions.

- Stratégies de « relation d'aide » à la por- critiques tée du médecin :

- discussion de cas en sous-groupe ;- illustration de la stratégie de chaque groupe sous forme de jeu de rôles.

- Les tâches du médecin en entrevue de "relation d'aide" :

- discussion à partir des lectures des participants.

- L'intervention du médecin, pas à pas... dans le but d'explorer l'affect du patient, sa logique interne, ses ressources et ses solutions :

- démonstration par les animateurs, ponctuée d'arrêts pour solliciter les suggestions des participants.

- Ce que je compte expérimenter avec mes patients : - tour de table.

\section{EXEMPLE 2}

Thème : violence familiale

- Le vécu de la violence conjugale et nos réactions comme intervenant :

- discussion après visionnement d'un stimulus vidéo.

-Violence : mythes, réalités et intervention :

- discussion du prétest complété par les participants ;

- questions-réponses à partir de lectures.

- Dépistage de la violence lors de la consultation médicale ponctuelle :

- jeu de rôles (visite à l'urgence d'une femme violentée, accompagnée de son conjoint).

- Les interventions aidantes du médecin de famille: - témoignage d'une patiente survivante de violence conjugale et échange avec les participants ;

- discussion de vignettes cliniques issues des incidents critiques (en sous-groupes et plénière).

- Rédaction d'un incident critique pour le prochain atelier. nent systématiquement les activités pédagogiques et les interventions d'animation jugées insatisfaisantes ou controversées. Pour ce faire, ils recueillent des informations issues de points de vue différents : celui des participants à qui sont soumis les changements au fur et à mesure pour les valider, celui des animateurs de l'atelier, et un regard extérieur obtenu auprès d'enseignants qui ne sont pas impliqués directement dans l'animation de l'atelier. Pour que cette cueillette de données s'intègre bien à l'activité, des outils très simples ont été utilisés, soit la formulation d'un commentaire (le verbatim étant noté lorsqu'il s'agit d'un commentaire verbal), et la cotation sur une échelle de 1 à 10 . Le moment et l'objet des différents commentaires recueillis, de même que les acteurs auprès de qui ils ont été recueillis, sont décrits au tableau 3.

L'analyse du contenu des différents commentaires est faite au moyen d'une discussion entre les auteurs qui recherchent surtout des précisions sur les embûches rencontrées, des réactions surprenantes et des opinions contradictoires. Ils poursuivent ensuite leur discussion conformément à la démarche décrite à la figure 1 pour chacun des ateliers. Ils sont alors à la recherche de stratégies correctrices qu'ils mettront à l'essai avec le groupe suivant de résidents. Les stratégies sont modifiées jusqu'à ce que, de l'avis des participants et des animateurs, elles ne créent plus d'embûches dans les séances et ce, avec des groupes dont le profil de participants est jugé varié.

Au cours des trois dernières années, la discussion entre les auteurs après chaque série d'ateliers a aussi porté sur les dénominateurs communs au niveau des stratégies pédagogiques qui ont dû être introduites ou modifiées depuis le début de la démarche. Ainsi, une catégorisa- 


\section{Recherche et Perspectives}

Figure 1 : cueillette et analyse de l'information

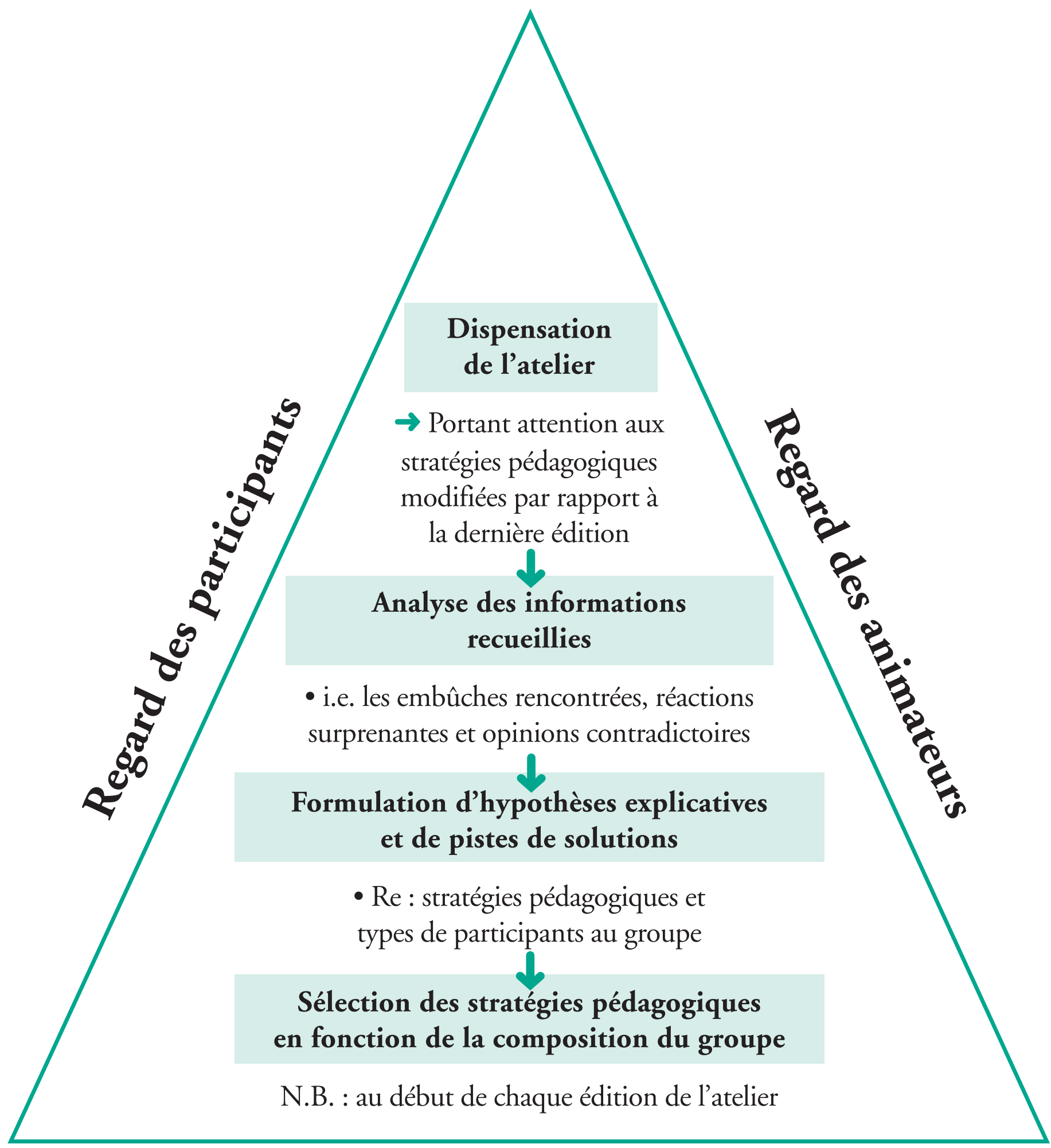

\section{Regard extérieur}




\section{Tableau 3 : trois regards sur l'animation}

\begin{tabular}{|c|c|c|}
\hline Regard des participants & Regard des animateurs & Regard « extérieur» \\
\hline $\begin{array}{l}\text { EN FIN D'ATELIER : } \\
\text { - Commentaires des participants, en } \\
\text { groupe, sur le déroulement (straté- } \\
\text { gies pédagogiques). } \\
\text { - Commentaires écrits de chaque } \\
\text { participant : cote de satisfaction de } \\
\text { l'atelier (échelle de } 1 \text { à 10) et for- } \\
\text { mulation du message principal } \\
\text { retenu. } \\
\text { APRÈS LA SÉRIE D'ATELIERS : } \\
\text { - Perception des acquis pour chacun } \\
\text { des thèmes en cotant individuelle- } \\
\text { ment (échelle de } 1 \text { à 10) la compé- } \\
\text { tence " avant " et " après " la série } \\
\text { d'ateliers. } \\
\text { - Suggestions pour améliorer la série } \\
\text { d'ateliers en regard des stratégies } \\
\text { pédagogiques (formulées lors de } \\
\text { l'entrevue individuelle). }\end{array}$ & $\begin{array}{l}\text { - Perception du style des participants } \\
\text { lors de l'entrevue individuelle en } \\
\text { début de semestre. } \\
\text { - Rencontre des deux animateurs } \\
\text { immédiatement avant et après } \\
\text { l'atelier pour discuter du fonction- } \\
\text { nement du groupe et de l'effet des } \\
\text { stratégies expérimentées. } \\
\text { - Consultation des incidents cri- } \\
\text { tiques des participants pour en } \\
\text { dégager le niveau des préoccupa- } \\
\text { tions du groupe par rapport au } \\
\text { prochain thème d'atelier. } \\
\text { - Rencontre semestrielle de l'équipe } \\
\text { d'animation, une fois la série d'ate- } \\
\text { liers complétée avec un groupe, } \\
\text { pour dégager les observations du } \\
\text { semestre, les évaluations formulées } \\
\text { par les participants et planifier de } \\
\text { nouvelles stratégies. }\end{array}$ & $\begin{array}{l}\text { - Consultation d'autres animateurs } \\
\text { membres de l'équipe au sujet d'un } \\
\text { atelier pour lequel ils n'ont pas été } \\
\text { impliqués comme animateurs.; à } \\
\text { propos des difficultés rencontrées } \\
\text { et des pistes de solution adoptées } \\
\text { en vue d'un nouvel ajustement des } \\
\text { stratégies. } \\
\text { - Analyse du processus d'animation } \\
\text { ainsi que du contenu des ateliers } \\
\text { par un enseignant d'une autre } \\
\text { unité s'étant joint à l'équipe d'ani- } \\
\text { mation en cours de processus. } \\
\text { - Consultation annuelle des anima- } \\
\text { teurs responsables d'enseignement } \\
\text { analogue dans les autres UMF du } \\
\text { programme à propos des stratégies } \\
\text { et difficultés rencontrées. } \\
\text { - Commentaires d'enseignants issus } \\
\text { de l'équipe régulière de supervision } \\
\text { de l'UMF (invités à titre de coani- } \\
\text { mateurs ponctuels à diverses occa- } \\
\text { sions pendant le semestre), sur nos } \\
\text { stratégies et l'impact sur le groupe } \\
\text { de participants. }\end{array}$ \\
\hline
\end{tabular}

tion de stratégies a été recherchée et construite progressivement. Parallèlement, l'importance d'effectuer une analyse des stratégies à privilégier en fonction du type de participants a émergé du processus et a été explorée. Les résultats de ces deux types d'analyse longitudinale sont présentés à la section suivante.

\section{Résultats \\ Difficultés et stratégies}

Les difficultés rencontrées et les solutions apportées ont permis d'identifier six catégories de stratégies pédagogiques : transformer des besoins démontrés en besoins ressentis ; amener les résidents à explorer leurs difficultés relationnelles avec les patients ; générer le goût d'une intervention guidée par des balises cognitives reconnues; faciliter le passage de la discussion à l'expérimentation concrète ; induire l'habitude de l'analyse réflexive en cours d'action et favoriser le transfert des apprentissages en groupe à la pratique clinique.

\section{Transformer des besoins démontrés en besoins ressentis.}

Certains résidents éprouvent des réticences à participer aux ateliers. Ils évoquent la priorité d'apprendre à gérer les situations biomédicales et le fait qu'au prégradué, ils aient discuté de la communication patient-médecin lors de la formation portant sur les habiletés cliniques. Ils surévaluent leurs acquis antérieurs et sont peu motivés à 


\section{Recherche et Perspectives}

approfondir le sujet. Ils n'en perçoivent pas la pertinence en ce début de résidence.

Notre stratégie vise à aider les participants à percevoir leurs acquis réels et leurs lacunes au plan de la communication patient-médecin et à ressentir leurs besoins de formation tout au long du programme. Quatre activités soutiennent cette stratégie : l'atelier d'introduction, la décision de s'engager personnellement dans le groupe, le partage des objectifs après cinq ateliers, et l'utilisation d'incidents critiques pour saisir la pertinence des ateliers. Dans un atelier d'introduction, le groupe identifie des situations cliniques difficiles vécues ou anticipées. A travers la discussion de leurs difficultés en groupe, ils expérimentent un aperçu du type et du climat d'échanges proposés durant les ateliers. Ils réalisent que savoir théoriquement quoi faire, face à une situation problématique n'est qu'un début dans l'acquisition du savoir-faire et qu'ils ont besoin d'expérimenter de nouvelles alternatives face aux diverses situations. Un lien est établi entre leurs difficultés et les thèmes des ateliers.

Par la suite, chaque participant est rencontré individuellement afin de préciser son choix de s'engager ou non à participer aux ateliers. Offrir ce choix aux résidents plutôt que de les obliger à y assister, comme ce fut le cas au cours des premières années du programme, les incite à s'y impliquer personnellement et à s'approprier la démarche. A l'occasion de l'entrevue individuelle, ils précisent les difficultés relationnelles qu'ils souhaitent corriger en groupe de même que certains moyens d'y parvenir. Sont également discutées leurs appréhensions par rapport aux jeux de rôles, aux activités d'apprentissage interactif et aux diverses modalités d'animation du groupe. Moins de $2 \%$ des résidents optent pour un cheminement individuel sous forme d'échanges écrits hebdomadaires avec un animateur sur les thèmes des ateliers. Cette politique de rencontres individuelles et d'engagement volontaire réduit significativement les réactions d'opposition dans le groupe. Au besoin, après cinq ateliers, les participants sont invités à reconsidérer leur décision et à ajuster leurs objectifs individuels. À ce moment, un temps est prévu pour que chaque résident expose au groupe sa démarche et reçoive des suggestions de moyens pour favoriser l'atteinte de ses objectifs.

À plusieurs occasions en fin de séances, les résidents décrivent des incidents critiques sur le thème du prochain atelier, c'est-à-dire des situations cliniques difficiles qu'ils souhaitent aborder différemment ${ }^{8}$. À travers les cas soumis, les animateurs décodent les lacunes des résidents par rapport au thème et planifient les façons d'y répondre au cours de l'atelier, soit en ajustant les objectifs d'apprentissage, soit en prévoyant des exercices ou discussions en sous-groupes pour traiter les nouveaux contenus identifiés. Ainsi, les incidents critiques serviront d'ancrages aux apprentissages.

\section{Amener les résidents à explorer leurs difficultés relationnelles avec les patients.}

Au cours de leur formation prégraduée, les résidents ont appris que la performance clinique biomédicale et technologique est très valorisée. Tout au long de leur formation médicale, ils sont souvent conditionnés à tout savoir et peu encouragés à reconnaître leurs difficultés de communication et à rechercher l'aide pour les résoudre.

La première heure de plusieurs ateliers est consacrée à des discussions sur des difficultés relationnelles rencontrées en clinique. Les résidents, et parfois les animateurs, soumettent alors quelques questions spécifiques à leurs collègues, lesquels agissent comme consultants ${ }^{9}$. Dans un premier temps, ceux-ci posent, à tour de rôle, une question de clarification. Puis, ils discutent de solutions alors que celui qui consulte le groupe, écoute en retrait les suggestions émises. Enfin, ce dernier livre au groupe ce qu'il retient et compte appliquer avec son patient. Au fil de ces discussions, la reconnaissance des difficultés relationnelles est valorisée, laquelle est la première étape du processus d'autorégulation constante caractérisant le fonctionnement en entrevue d'un expert. Bref, au lieu de taire leurs difficultés, les résidents sont encouragés à les discuter avec le groupe. Au cours des six mois de stage, chaque résident consultera ses collègues une à deux fois et les informera du suivi des recommandations qu'il aura appliquées avec son patient.

Tout au long des ateliers, à travers les discussions et les diverses mises en situations concrètes, les résidents apprennent que s'ouvrir à parler de problèmes ne signifie pas pour autant être un intervenant à problèmes, mais plutôt qu'il s'agit d'une condition de base pour être un clinicien efficace. La reconnaissance de leurs difficultés relationnelles et les opportunités à les dénouer dans le groupe, en se plaçant à tour de rôle en position de patient et de médecin durant les exercices pratiques, les aident à améliorer leurs habiletés de communication empathique avec leurs patients. 


\section{Générer le goût d'adopter une intervention guidée par des balises cognitives reconnues.}

Les premières réactions des résidents face à leurs difficultés relationnelles avec les patients sont souvent émotives, reflétant les caractéristiques individuelles de chacun. Les participants se rendent compte des limites d'un fonctionnement par essai-erreur et recherchent des principes d'intervention efficace avec leurs patients.

Au cours des ateliers, les animateurs fournissent aux résidents des stratégies spécifiques de résolution de problèmes inspirées des découvertes de la psychologie cognitive : utiliser les apprentissages issus des expériences antérieures, tenir compte du contexte particulier de la consultation, dégager les patterns répétitifs de comportements qui posent problème, outiller et soutenir la personne qui consulte par rapport au changement, favoriser le transfert de ses apprentissages dans la vie quotidienne, etc. Cette approche dite "stratégique » fournit aux apprenants des balises cognitives reconnues et validées (concepts-clés, grilles d'entrevue, etc.) ${ }^{10}$. A cet effet, en faisant les lectures recommandées pour préparer l'atelier, les résidents sont invités à rechercher des éléments de solution par rapport à une situation clinique problématique.

Pour la partie de l'atelier intitulée retour sur les textes, les animateurs insistent sur trois types de questions des participants : les surprises, les clarifications et les désaccords, tout en ayant le souci d'établir des liens avec la discussion de cas qui a précédé. Ils aident ainsi le groupe à saisir les implications concrètes des concepts-guides d'intervention efficace proposés dans les textes. Ces balises cognitives deviennent les consignes d'observation pour les jeux de rôles et autres exercices.

Résoudre des difficultés de communication en s'inspirant de concepts valides donne au participant le goût de chercher, de lire et de discuter de solutions au lieu de se limiter à des réactions interpersonnelles improvisées avec ses patients.

\section{Faciliter le passage de la discussion à l'expérimenta- tion concrète.}

L'apprenant qui souhaite trouver rapidement des options d'intervention à ses difficultés de communication, risque de se limiter à des discussions intellectuelles et superficielles. Les animateurs privilégient une mise à l'essai concrète des alternatives suggérées. Une participation active est favorisée par deux modalités d'animation : le jeu de rôles et les témoignages d'un patient interviewé par le groupe. L'engagement initial des résidents à travailler une habileté de communication concrète, à travers leur participation dans le groupe, les responsabilise par rapport au déroulement des ateliers. Les participants sont initiés progressivement à l'utilisation des jeux de rôles. Durant les premiers ateliers, les animateurs font des démonstrations de portions d'entrevues et les résidents sont appelés à formuler des options d'intervention lors d'arrêts aux moments-clés de l'entretien. Par la suite, les participants sont sensibilisés aux jeux de rôles " collectifs " où ils interviennent alternativement comme médecins auprès d'un patient joué par un animateur. Enfin, les résidents s'impliquent tour à tour comme patients ou médecins dans des jeux de rôles traditionnels à l'aide de canevas d'interventions efficaces. Les règles d'animation d'un jeu de rôles favorisant l'apprentissage sont respectées : la préparation ou mise en contexte, le jeu de rôles lui-même et la phase de retour $^{11}$. Les animateurs aident le groupe à identifier et à résoudre les impasses relationnelles. Lorsqu'ils sont habitués aux jeux de rôles, les résidents sont invités à jouer brièvement le rôle de leur patient suite à une discussion de cas dans le but de tester l'application de certaines suggestions retenues. Des options d'interventions sont parfois illustrées à partir de segments d'entrevues réalisées par les animateurs ou les résidents.

A quelques occasions, un patient est invité, à titre d'expert, à rencontrer le groupe et à livrer un témoignage touchant sa problématique spécifique (ex. : divorce, violence conjugale, deuil, etc.). De cette façon, l'atelier devient le théâtre de la vie professionnelle où, confrontés à divers scénarios cliniques posant problème, les résidents testent des questions qu'ils ne risqueraient pas d'emblée avec leurs patients. Ils pourront, par la suite, les adapter à leur pratique clinique.

\section{Induire l'habitude de l'analyse réflexive en cours d'action.}

Il arrive que l'intérêt des participants fluctue vis-à-vis des ateliers. Lorsqu'une majorité de participants semble désinvestir les ateliers, l'animation pose un défi certain. Une telle impasse survenant dans le groupe est considérée de la même façon qu'une impasse dans la relation patient-médecin. Cette analogie est explicitée aux participants. La résolution des impasses relationnelles avec les patients est souvent l'occasion d'ajustements béné- 


\section{Recherche et Perspectives}

fiques au processus thérapeutique. De même, la gestion d'impasses dans le groupe permet des adaptations favorables à l'évolution du groupe.

En présence de telles impasses, il importe donc d'annoncer une pause dans le déroulement de l'atelier et d'amorcer une démarche réflexive sur le fonctionnement du groupe. Les animateurs s'impliquent alors comme membres actifs du groupe en énonçant le problème tel qu'ils le vivent. Par exemple, dans le cas de la fluctuation de l'intérêt vis-à-vis des ateliers, ils décrivent leur malaise, questionnent ce désinvestissement, puis aident à la clarification des choix quant aux apprentissages visés et au fonctionnement ultérieur du groupe. Ils encouragent la discussion ouverte et respectueuse comme force autorégulatrice pour la croissance du groupe et l'implication des participants.

Pour désamorcer précocement l'éventualité d'impasses relationnelles, il est utile de prévoir des moments réguliers de réflexion par rapport à ce qui est vécu. Des mises à jour périodiques permettent de clarifier les malentendus au niveau des interactions et d'ajuster les ateliers aux besoins réels des participants. De plus, lors de l'entrevue individuelle finale, un retour est effectué sur leur appréciation de l'animation du groupe.

Par le souci constant de démarche réflexive, les animateurs appliquent leur cadre d'analyse à l'animation. Ils font des liens entre ces mises au point dans le groupe et l'importance de répéter un processus similaire en clinique. Par exemple, en situation d'inobservance d'un patient, il convient de discuter avec lui de ce qui se passe et de négocier de nouvelles ententes de collaboration ${ }^{12}$. Cette étape d'application de la démarche réflexive à une situation clinique s'avère essentielle pour ancrer le processus et son utilité chez les participants.

\section{Favoriser le transfert des apprentissages en groupe à la pratique clinique.}

Pour appliquer ce qu'ils ont appris, les résidents ont besoin d'encadrement au moment des supervisions de leur pratique quotidienne : ce suivi est primordial. Pour assurer cette continuité, plusieurs stratégies sont utilisées. Certains superviseurs participent à un ou quelques ateliers pour en connaître le contenu en détails. Les messages principaux des ateliers sont résumés au cours de rencontres de superviseurs. Lors d'une formation pédagogique en groupe sur le feedback constructif, des exemples de façons d'intégrer ces concepts à la supervi- sion quotidienne sont présentés et discutés. Finalement, les animateurs informent régulièrement les superviseurs du cheminement des résidents dans le groupe.

Les supervisions directes et les discussions de cas permettent de vérifier et de compléter l'intégration des acquis. C'est en voyant la pertinence des enseignements au quotidien que les résidents se les approprient ; c'est en multipliant les occasions et les sources de feedback cohérent et complémentaire que l'intégration se réalise. Le visionnement conjoint, résident-superviseur, de vidéos d'entrevues et la pratique d'entrevues médicales simulées avec des superviseurs dans le rôle de patients aident aussi les résidents à identifier et à combler certaines lacunes moins évidentes dans leur fonctionnement.

L'entrevue individuelle finale est l'occasion de revoir le cheminement des participants et de discuter de leur autoévaluation. En retour, les animateurs leur donnent un feedback personnalisé sur leurs acquis et ce qui leur reste à travailler en regard de leurs objectifs initiaux.

\section{Stratégies privilégiées selon le type de participants}

De même que le changement en psychothérapie est surtout attribuable au patient lui-même, l'évolution des apprentissages en petit groupe de formation nous apparaît fortement liée aux participants en présence. L'analyse de la description spontanée des participants faite par les animateurs au cours des dix dernières années permet de dégager trois principaux types d'apprenants. L'identification de ces différents types peut permettre de privilégier des catégories de stratégies plus ciblées. Le type A est l'apprenant ouvert qui se reconnaît d'emblée dans l'approche globale, systémique et négociée. Confiant et réaliste, il identifie adéquatement ses forces et ses limites. L'apprenant de type B, " pratico-pratique » et hésistant, recherche des " trucs " concrets pour composer avec les situations cliniques qui lui posent problème. Il tend à se déprécier lorsqu'il s'autoévalue. Le type $\mathrm{C}$ adhère à une vision biomédicale stricte et s'oppose à une approche davantage centrée sur le patient. Il surévalue ses habiletés et acquis relationnels; ses capacités d'écoute et de respect de l'autre sont souvent limitées. Selon le type de résident dominant dans le groupe, soit par le nombre, soit par le leadership ou l'influence qu'un individu exerce, les animateurs auront à privilégier certaines catégories de stratégies d'animation pour maximiser les apprentissages du groupe. Le tableau 4 
résume les catégories de stratégies qui ont été privilégiées jusqu'à maintenant pour personnaliser l'animation en fonction du type dominant dans le groupe.

Lorsque des apprenants de type A sont dominants dans le groupe, leur intérêt marqué et leur facilité à reconnaître leurs difficultés relationnelles avec les patients les amènent à les travailler avec leurs collègues. Ils démontrent, par leur propre exemple, qu'il s'agit d'une force et non d'une faiblesse et encouragent ainsi les autres à s'exposer dans le groupe. Ils sont, d'emblée, volontaires pour s'impliquer dans les jeux de rôles et autres exercices d'apprentissage. Enfin, ils prennent goût rapidement à réfléchir sur leur action pour dénouer une impasse relationnelle et offrent spontanément leur aide lorsque les animateurs procèdent à l'analyse réflexive d'une situation-problème dans le groupe.

Par ailleurs, lorsque des apprenants de type B sont plus nombreux ou exercent un leadership dans le groupe, ils recherchent d'abord des trucs concrets pour les patients qui leur causent problème. Les animateurs auront avantage à leur démontrer rapidement l'utilité de balises cognitives concrètes et simples pour guider leurs interventions cliniques de façon à maintenir leur motivation. Ainsi, ils apprennent graduellement à combler leurs lacunes au contact de situations cliniques difficiles et à y trouver un bénéfice. Toutefois, ils demeurent dépendants du contexte pour poursuivre leurs apprentissages. Avec eux, il importe d'assurer le suivi des acquis amorcés dans le groupe au niveau de la supervision au quotidien et ce, tout au long de leur formation. La supervision directe s'avère particulièrement utile pour faire des liens entre ce qu'ils rencontrent comme difficulté en clinique et ce que les ateliers proposent.
En revanche, lorsque des apprenants de type $\mathrm{C}$ exercent une influence prépondérante dans le groupe, ils tendent à s'opposer au nouveau modèle proposé et refusent de questionner leur façon de voir en présence de leurs collègues. Lorsqu'ils réussissent en entrevue individuelle à identifier certaines lacunes relationnelles, il y a des possibilités d'entente sur des objectifs spécifiques à travailler en groupe. Lorsqu'ils décident de le faire, ils s'impliquent volontiers dans les jeux de rôles et autres exercices concrets. Ils éprouvent des difficultés à demeurer actifs dans le groupe et apprécient des consignes précises, surtout en situation d'observateurs. Les animateurs doivent éviter de les confronter directement dans le groupe, car ils tendent alors à durcir leurs positions. Enfin, il importe de s'entendre explicitement avec eux sur les liens entre ce qu'ils travaillent dans le groupe et dans leurs supervisions quotidiennes. Un travail de collaboration étroite avec leurs superviseurs est nécessaire pour les aider à cibler ce qui pose problème dans leur fonctionnement et les encourager à y travailler.

\section{Discussion}

La démarche effectuée possède des caractéristiques qui supportent la validité des résultats présentés et en situe les limites ${ }^{13,14}$. Les observations ont porté sur tous les groupes de participants à l'activité sur une longue période, maximisant les chances de voir se manifester un éventail de réponses variées. Les données recueillies ont fait appel à une triangulation des observations permettant de cerner le phénomène à l'étude selon divers points de vue, même s'il n'y avait pas de réels observateurs externes du processus. La validation régulière auprès des participants et le fait que les chercheurs

\section{Tableau 4 : catégories de stratégies à privilégier en fonction du type d'apprenants}

\begin{tabular}{|l|c|c|c|c|c|c|}
\hline $\begin{array}{r}\text { Catégories } \\
\text { de stratégies }\end{array}$ & $\begin{array}{c}1 . \\
\text { Besoins }\end{array}$ & $\begin{array}{c}2 . \\
\text { Intros- } \\
\text { pection } \\
\text { d'apprenants }\end{array}$ & $\begin{array}{c}3 . \\
\text { Balises }\end{array}$ & $\begin{array}{c}4 . \\
\text { Expéri- } \\
\text { mentation }\end{array}$ & $\begin{array}{c}\text { Analyse } \\
\text { réflexive }\end{array}$ & $\begin{array}{c}6 . \\
\text { Transfert }\end{array}$ \\
\hline Type A & $* *$ & $*$ & $*$ & $*$ \\
\hline Type B & $*$ & & $* *$ & & $*$ \\
\hline Type C & $* *$ & & & $*$ & & $*$ \\
\hline
\end{tabular}

Légende : **Première catégorie de stratégies à privilégier

*Autres catégories de stratégies à privilégier 
demeurent acteurs du phénomène à l'étude ont toujours été au centre de la démarche. La collecte et l'analyse des données ont été effectuées en parallèle et de façon itérative, conduisant à une catégorisation de stratégies intimement liée aux observations faites sur le terrain. Pour ces raisons, la structure des stratégies pédagogiques qui en résulte pourra guider efficacement le travail des auteurs avec les prochains groupes, et même faciliter un enseignement analogue dans d'autres milieux. L'impact des interventions favorisant le transfert des apprentissages faits en groupe à la pratique clinique (sixième stratégie) reste à tester davantage.

Le processus d'analyse et les choix de stratégies pédagogiques sont teintés par l'adhésion des auteurs aux principes de l'approche systémique et centrée sur le patient et, par extension, de l'enseignement centré sur l'apprenant. Les stratégies élaborées sont congruentes avec les principes andragogiques, tout en y ajoutant des mises en application concrètes, issues de l'analyse de l'expérience auprès de petits groupes ${ }^{15}$.

En ce qui concerne les stratégies à privilégier en fonction du type de participants dans le groupe, il s'agit d'une hypothèse qui a émergé de l'expérience. L'analyse devra être poursuivie pour vérifier si leur utilisation permet d'influencer le processus d'apprentissage des participants.

\section{Conclusion}

En plus de permettre de dégager des catégories de stratégies d'enseignement en groupe des habiletés de communication patient-médecin-famille, la démarche de l'étude qui s'appuie sur l'analyse réflexive, a supporté la motivation des enseignants impliqués. Elle leur a permis de profiter des difficultés rencontrées comme des occasions d'adapter constamment leurs stratégies pédagogiques aux nouvelles découvertes et aux suggestions des participants et du milieu. Suite à dix années d'expérimentation et même si le processus se poursuit, les auteurs ont jugé important de procéder à cette synthèse des données analysées. Ce fut l'occasion de dresser une liste des prochaines pistes d'exploration : rendre les stratégies plus opérationnelles pour d'autres animateurs, approfondir les moyens pour favoriser le transfert des apprentissages en groupe à la pratique clinique et confronter au test de la réalité l'application du modèle de stratégies à privilégier selon les types de participants. Bref, l'analyse réflexive continuera à rendre l'expérience d'enseignement vivante, dynamique et sans cesse renouvelée.

\section{Références}

1. Stewart MA. Effective Physician-Patient Communication and Health Outcomes, A review. Can Med Assoc 1995 ; 152 : 1423-1413.

2. Christie-Seely J. Working with family in primary care: A system approach to Health and illness. New-York : Praeger. 1984.

3. Boulé R, Girard G. L'approche centrée sur le patient : concepts et exemples. Revue de la Médecine Générale 1999; 166 : 347-381.

4. Stewart MA, Brown JB, Weston WW, McWinney IR, McWilliam CL, Freeman TR. Patient Centered Medicine. Transforming the Clinical Method. Thousand Oaks : Sage Publication 1995.

5. Côté L, Clavet D, St-Hilaire S, Vaillancourt G, Blondeau F, Martineau B. La relation médecinpatient en médecine familiale, une expérience de planification d'activités d'apprentissage. Can Fam Physician 1999 ; 45 : 1250-54.

6. Boulé R, Girard G. Une expérience d'enseignement de la relation médecin-malade. Psychologie Médicale 1991 ; 23 : 637-9.

7. St-Arnaud Y. Connaître par l'action. Montréal : P.U.M 1992.

8. Flanagan J.C. The critical incident technique. Psychol Bull. 1954 ; 51 : 327-358.

9. Andersen T. The reflecting team and meta-dialogues in clinical work. Family Process 1987 ; $26: 415-28$.

10. Tardif J. Pour un enseignement stratégique, Montréal : Édition Logiques 1992 ;295-378.

11. Wohlking W, Gill PJ. Role Playing, Englewoods, NJ : Educational Technology Publications 1980 ; 23-49.

12. Girard G, Grand'Maison P. L'approche négociée, modèle de relation patient-médecin. Le Médecin du Québec 1993 ; 12 : 31-39.

13. Mays N, Pope C. Assessing quality in qualitative research, BMI 2000 ; 320 : 50-52.

14. Poupart J, Deslauriers JP, Groulx LH, Lafrenière A, Mayer R, Pires AP. La recherche qualitative. Enjeux épistémologiques et méthodologiques. Montréal : Gaëtan Morin 1997.

15. Knowles $M$. The Adult Learner : A neglected species. Houston : Gulf 1984. 